typeset using JPSJ.sty $<$ ver.1.0b $>$

\title{
Physical Validity of Assumptions for Public Exposure to Mobile Phones
}

\author{
Tsuyoshi Hondou* \\ Department of Physics, Tohoku University, Sendai 980-8578, Japan
}

(Received )

\begin{abstract}
KEYWORDS: free energy, boundary condition, non-thermal interaction, mobile phone, public safety, precautional principle, security hole
\end{abstract}

In the Letter, ${ }^{1)}$ we derived an analytical formula which estimates the level of public exposure to electromagnetic waves in closed areas. This is the first study in which one approximately predicts how much the exposure level increases by using two indispensable factors; 1) reflection of the electromagnetic waves at the boundary and 2) additivity of emissions. In their Comment, Kramer et al. claimed that public exposure in closed areas does not impose additional health risks in comparison with those in any other location. Their claim was not toward the analytical derivation of the exposure level, which is the primary result of the Letter. However, we found that the Comment was based on several implicit assumptions which were not relevant to the issue being addressed and thus the claim itself was not valid. Some of the assumptions were based on improper applications of the fundamentals of physics. In this Reply, we will clarify such misunderstandings arising in the Comment through careful consideration of their implicit assumptions. The readers will find the essential issues which should always be taken into account.

First, we wish to reconfirm the absolutely essential requirements to deal with issues of public safety. To prevent security holes in public safety, one is required to have a sufficiently deep insight into all the issues concerned, because a one-sided way of looking at things may cause unexpected problems. It would be best to start discussions from the fundamentals of physics which relate to the issues. A confusion between two distinct physical quantities can often be seen in the studies of exposure, where electromagnetic energy is often equated with thermal energy. As a fundamental fact, it is known that the energy of an electromagnetic wave is different from thermal energy, as immediately proved by the second law of thermodynamics. ${ }^{2)}$ Thermodynamics reveals that any physical system can develop autonomously only in the direction in which the entropy of the system increases. One can fully change the energy of an electromagnetic wave into thermal energy, however the reverse is impossible. ${ }^{3)}$ It is a universal fact that the status of the energy of an electromagnetic wave is much more coherent than that of thermal energy. The difference in energy can be described as, free energy or entropy. ${ }^{4)}$ We can learn from this consideration that it is a natural phenomenon that electromagnetic waves often interfere with living things

*E-mail address: hondou@cmpt.phys.tohoku.ac.jp to a much large extent than does thermal energy under exposure to heat even if the apparent quantities of energy are the same.

It is known that there are two kinds of electromagnetic interference for humans caused by electromagnetic waves, 1) thermal effect (heating) which is attributed to an increase in temperature as a result of the dissipation process of electromagnetic waves in the human body, and 2) non-thermal effect which is attributed to the property of the electromagnetic wave itself. The quantity Kramer et al. referred to in their Comment when they discussed safety issues was the specific absorption rate (SAR) which concerns the thermal effect; however, the non-thermal effect is indeed indispensable and must be considered, as stated in the last paragraph. For example, electromagnetic waves may interfere with the cardio-pacemakers implanted in cardiac patients. In the worst case of the interference, the person may pass away when such an interference continues for several minutes. Note that a legal medical issue remains that no evidence would exist after the interference disappears. The authors of the Comment completely ignored these serious issues, whereas the issue is explicitly stated in the Letter. The interference with pacemakers is reported to occur within a maximum distance of $30 \mathrm{~cm}$ between a mobile phone and pacemaker, ${ }^{5)}$ even though neither reflectivity of the boundary nor additivity of multiple phones has been taken into account in this experiment. In fact, the interference with pacemakers may occur even if the SAR limits (basic restriction by ICNIRP) for environmental exposures, an issue raised by the authors of the Comment, are cleared. This fact is also explicitly stated in the section "Purpose and Scope" of the document by ICNIRP that Compliance with the present guidelines may not necessarily preclude interference with, or effects on, medical devices... . Interference with pacemakers may occur at levels below the recommended reference levels. Advice on avoiding these problems is beyond the scope of the present document.... (see Ref. 2 of the Comment). It is obvious that the interference with pacemakers is caused by non-thermal interference, the mechanism of which is physically different from the thermal effect regulated by SAR criteria. The interference with hearing aids is also an example of current-day problems, where sufferers cannot use their hearing aids in certain places including commuter trains due to insufferable noise caused by the electromagnetic interference. Consideration of these 
examples reveals the limitation of the validity of the SAR criteria. SAR criteria are only applicable for recognized thermal effects and thus are not relevant to cover whole aspects of the safety issues by itself.

The above discussion has already proved that the central claim of the Comment is not valid. However, there remain crucial issues to be resolved for future studies. The authors of the Comment introduced the present SAR criteria without any consideration of the limitation of validity. What is more important for scientists is not the value of the criteria themselves but their scientific basis, because such criteria can hardly be adopted without arbitrary factors. The criteria were derived through the review of extensive studies. As stated in reference 7 , the basic restriction of ICNIRP (for microwave region) is based only on the study on the thermoregulatory response caused by the thermal absorption of electromagnetic waves. There are several other aspects regarding the origin of the problem even within the thermal effects. It should also be noted that the exposure level is often much lower than the well-recognized thermal effect in case of non-thermal interaction, which includes not only the interference with electromedical instruments but also the interference with living organisms themselves (biological effect). SAR criteria of the basic restriction were, however, based only on clear evidence at the time of submission (see, ref. 7). A number of studies, which had uncertainties in their results at the time, were not adopted in the basic restriction. Some of them reported adverse biological effects at a level much lower than that adopted for the basic restriction. However such not-well-recognized phenomena including nonthermal effects, were not considered even as "factors" to strengthen the basic restriction of ICNIRP, although there is a gap between the extent of our knowledge and what is actually occurring. Namely, in a scientific sense, the present SAR limit (basic restriction) does not guarantee that public safety is satisfied under the regulation. This background justifies the "precautional principle" adopted in European countries where more strict regulations of the exposure level than that of ICNIRP have been adopted in order to compensate for our ignorance on matters related to nature.

Furthermore, there are more implicit assumptions made in the discussion in the Comment, which should have been explicit: (I) The estimation of the SAR value $(25 \%)$ was made based on the assumption of equal thermal absorption for all persons: They neglected the "focus" phenomenon that the intensity of the wave can be concentrated due to the geometry of the reflective boundary condition. An example of this phenomenon can be found in a concert hall, where the loudness of the sound is often concentrated at special locations (seats) due to the reflection of the sound. This is a universal and essential physical property of waves. (II) Square distance decay of energy flux was assumed: It is among the fundamental requirements that we have to specify a boundary condition to solve any problem of electromagnetism. ${ }^{6)}$ Without a boundary condition, we cannot obtain a solution for electromagnetism. The authors seem to have implicitly assumed a free boundary condition for these prob- lems, as the boundary condition was not specified in their self-reference (Ref. 4) of the Comment. The boundary condition of systems considered in the Letter is, however, completely different from those in which a free boundary condition is appropriate. It is explicitly emphasized in the Letter that "short-range interaction paradigm" is no longer appropriate. (III) Mobile phones were assumed only to be used close to the head: This assumption is not always appropriate. People sometimes use mobile phones away from the head and close to stomach when they use an internet service such as $i$-mode; furthermore the users may include pregnant women. (IV) The number of mobile phones per person was assumed to be one: In several countries including Japan, many people possess multiple phones, for example, one for personal use and the other for business use. We have to consider the fact that the situation is changing and is no longer the conventionally assumed one. (V) A similar condition of human health was implicitly assumed between a person who uses a mobile phone and a person who suffers from interference: The user uses a mobile phone at his/her own risk for the sake of convenience. If he/she feels an adverse health condition, he/she can stop using the phone. However, a person who is exposed to the interference cannot control the existing exposure by him/herself. The person may be equipped with a pacemaker, for example. Exposure to general public must be considered separate from that to the users themselves.

As discussed above, the Comment were based on naive implicit assumptions which are neither relevant nor valid and sometimes with fatal consequences. This leads to several security holes in the regulation of public safety. Therefore the central claim of the Comment should be rejected. We hope that this correspondence between the Comment and the Reply can aid the reader in understanding the present issues and can contribute to future studies.

1) T. Hondou: J. Phys. Soc. Jpn. 71 (2002) 432

2) For example, H. Callen: Thermodynamics (John Wiley \& Sons, New York, 1960).

3) In this paper, we assume that the frequencies of electromagnetic waves are within a certain range. As found in black body radiation, thermal energy can easily be converted into electromagnetic energy. However, electromagnetic waves emitted from the black body is qualitatively different from the electromagnetic waves considered in this paper. Black body cannot emit coherent electromagnetic waves with special frequency. The difference can be described by entropy.

4) An analogous example is found in molecular motors, by replacing electromagnetic energy with chemical energy. Chemical energy can be converted into work with a $100 \%$ conversion rate, however thermal energy cannot be. See, for example, F. Jülicher, A. Ajdari and J. Prost, Rev. Mod. Phys. 69, (1997) 1269.

5) Fuyōdenpataisakukyōgikai (Electromagnetic Compatibility Conference Japan), Keitaidenwatanmatsutōno Shiyōnikansuru Chōsahōkokusho (Guidelines on the Use of Radiocommunication Equipment such as Cellular Telephones-Safeguards for Electronic Medical Equipment-) (Association of Radio Industries and Businesses, Tokyo, 1997) [in Japanese].

6) For example, W. K. H. Panofsky and M. Phillips: Classical Electricity and Magnetism (Addison-Wesley, Cambridge, 1961). 
7) International Commission on Non-Ionizing Radiation Protection, Guidelines for Limiting Exposure to Time-Varying Electric, Magnetic, and Electromagnetic Field (ICNIRP, Oberschleissheim, 1998). 